\title{
Direct Synthesis of Single-Walled Carbon Nanotubes on Silicon and Quartz-Based Systems
}

\author{
Yoichi Murakami, Shohei Chiashi, Yuhei Miyauchi and Shigeo Maruyama* \\ Department of Mechanical Engineering, The University of Tokyo, \\ 7-3-1 Hongo, Bunkyo-ku, Tokyo 113-8656, Japan
}

A newly developed technique of synthesizing single-walled carbon nanotubes (SWNTs) directly on the surface of $\mathrm{Si}$ and quartz substrates is introduced in this report. This technique adopted a liquid-based dip-coating method to mount a very small amount of catalyst metals on the surface of substrates using Mo/Co bimetallic acetate solution. The merits of this approach lie in its easy, costless, and geometry-flexible nature compared with conventional sputtering and deposition approaches. We used the alcohol catalytic chemical vapor deposition (ACCVD) method that can produce relatively high-quality SWNTs even at low temperatures down to $600^{\circ} \mathrm{C}$. This low-temperature process contributes to the prevention of the agglomeration of catalytic metals on the surface and chemical reaction between catalytic metal and silicon, which helps us to eliminate any kind of intermediating support materials. Thereby synthesized SWNTs on $\mathrm{Si}$ and quartz substrates under various CVD conditions are characterized by means of SEM, TEM, Raman scattering, and optical absorbance measurements. The underlying reasons our experimental procedure and choice of catalyst worked for the synthesis of SWNTs are discussed through comparative studies. At the end of this report, some possible applications of this technique are stated.

KEYWORDS: Carbon nanotubes, CVD, alcohol CCVD, silicon, direct synthesis.

\footnotetext{
* Corresponding author: maruyama@photon.t.u-tokyo.ac.jp
} 


\section{Introduction}

The discovery of single-walled carbon nanotubes (SWNTs) in 1993 by Iijima and Ichihashi ${ }^{1)}$ has thus far motivated numerous researchers in various fields trying to utilize the remarkable physical properties $^{2)}$ of this newly found 1-D carbon allotrope. Experimental studies on SWNTs have become possible since the discoveries of laser-furnace ${ }^{3)}$ and arc-discharge ${ }^{4)}$ techniques to produce bulk amounts of SWNTs, and the more scalable CVD technique ${ }^{5-12)}$ that has now become dominant in the mass synthesis of SWNTs.

In attempting to fabricate SWNT-based devices, an appropriate incorporation of SWNTs into silicon or other material-based systems is strongly desired. Thus far, several studies have been reported in which carbon nanotubes were synthesized on the substrates using various approaches. Kong et al. ${ }^{13)}$ and Franklin et al. ${ }^{14)}$ spun-coated their Fe/Mo catalyst with alumina support particles to form patterned catalytic islands on Si surfaces and synthesized small amounts of SWNTs. Cassell et al. mounted mesoporous alumina/silica on Si substrates to support their Fe/Mo catalyst and generated SWNTs ${ }^{15)}$. In other studies, the mesoporous silica was coated on a Si substrate in the form of a thin film as a supporter, leading to the production of MWNTs ${ }^{16-18)}$ or SWNTs ${ }^{19}$. Although it depends on the assumed application, the support material is sometimes undesired because this is an additional material that is not essential for the functioning of the devices. One way to circumvent this is presented by Li et al. ${ }^{20)}$ who did not use a support material but utilized ferritin protein for sparsely located iron nanoparticles on a flat $\mathrm{SiO}_{2}$ substrate to synthesize SWNTs. Homma et al. ${ }^{21)}$ dispersed commercially available $\mathrm{Fe}$ or $\mathrm{Fe}_{2} \mathrm{O}_{3}$ nanoparticles in ethanol and loaded them on a Si substrate. They produced thin carbon nanotubes including SWNTs at $950^{\circ} \mathrm{C}$ with the catalyst of $\mathrm{Fe}_{2} \mathrm{O}_{3}$, though the selectivity to SWNTs is unknown.

All studies referred above ${ }^{13-21)}$ used liquid-based approaches for their catalyst preparation; however, in some cases, catalytic metals were mounted on substrates using dry processes such as sputtering or deposition ${ }^{22-27)}$. Although selective synthesis of SWNTs on a flat Si surface was reported recently ${ }^{27}$, these methods are prone to forming relatively large (i.e. more than $10 \mathrm{~nm}$ ) agglomerates of catalyst metals and to producing multi-walled carbon nanotubes (MWNTs). For examples, the dominant products reported by Nerushev et al. ${ }^{22)}$ and Yoon et al. ${ }^{23)}$ were MWNTs, though they reported the synthesis of SWNTs. Delzeit et al. ${ }^{24)}$ synthesized SWNTs with relatively high quality and selectivity by pre-depositing $20 \mathrm{~nm}$ of an $\mathrm{Al}$ underlayer on a Si surface before depositing $1 \mathrm{~nm}$ of Fe/Mo catalyst. However, such an underlayer resulted in significant surface roughness due to an agglomeration of $\mathrm{Al}$ caused by the heat during CVD. A similar attempt was made by Zhang et al. ${ }^{25)}$ but this was also accompanied by an agglomeration of an $\mathrm{Al}$ underlayer. Although no SWNTs were produced in the case of a Si substrate, Hongo et al. ${ }^{26)}$ sputtered Fe on the A-face of a sapphire crystal and synthesized a 
relatively abundant amount of SWNTs at the CVD temperature of $800^{\circ} \mathrm{C}$. In other reports SWNTs were successfully incorporated on a catalyst-patterned substrate as a field-emission transistor (FET) ${ }^{28,29)}$, as a different approach from conventional post-dispersion of bulk-produced SWNTs before placing electrodes $^{30)}$. In their prototypes, a very low (one or slightly more) number of SWNTs were grown from catalytic islands of much larger dimensions $\left(3 \mu \mathrm{m}^{28)}\right.$ and $100 \mu \mathrm{m}^{29)}$ ), indicating that there were very few active sites and most catalyst areas did not participate. It is desirable to raise the area efficiency of the catalyst for future downsizing and increasing the concentration of FET density.

We here report a technique of synthesizing SWNTs directly onto the surface of Si and quartz flat surfaces with some discussions on our choice of CVD procedure and bimetallic catalyst. In the proposed technique, a small amount of metal catalyst is loaded through dip coating of a Mo/Co acetate solution, by which a very fine dispersion of bimetallic catalyst of approximately 1-2 nanometers in size is obtained. The alcohol CCVD technique ${ }^{11,12)}$ that can generate relatively high-quality SWNTs even at a low temperature of $600^{\circ} \mathrm{C}^{11)}$ was used in order to minimize any side effect caused by the high temperature in the CVD process, as discussed later.

\section{Experimental}

A detailed description of the catalyst loading process is presented in our previous report ${ }^{31)}$. In short, a metal acetate solution is prepared by adding molybdenum acetate $\left(\mathrm{CH}_{3} \mathrm{COOH}\right)_{2}$ Mo and cobalt acetate $\left(\mathrm{CH}_{3} \mathrm{COOH}\right)_{2} \mathrm{Co}-4 \mathrm{H}_{2} \mathrm{O}$ into ethanol so that the concentration of each metallic species over the total solution is $0.01 \mathrm{wt} \%$. The mixture is then sonicated for $2 \mathrm{~h}$ to dissolve these acetic salts. A substrate piece, appropriately sized, is vertically suspended by a clip connected to a nylon string in the metal acetate solution for $10 \mathrm{~min}$, and then the substrate is drawn-up from the solution at a constant speed of 4 $\mathrm{cm} / \mathrm{min}$. Immediately after this, the piece is placed in a furnace maintained at $400^{\circ} \mathrm{C}$ for $5 \mathrm{~min}$ in order to convert metal acetate into metal oxides of bimetallic Mo/Co catalyst on the surface.

The schematic of our CVD apparatus is shown in Fig. 1. It is composed of an ethanol reservoir, a quartz tube (26 mm i.d., $1 \mathrm{~m}$ long), an electric furnace, and a rotary vacuum pump. In the CVD process, the catalyst-loaded substrate is placed on a quartz boat that is located at the central position of the quartz tube as shown in Fig. 1. While the furnace is being heated, approximately $300 \mathrm{sccm}$ of $\mathrm{Ar} / \mathrm{H}_{2}$ gas (3 \% $\mathrm{H}_{2}$ ) is made to flow with only the 'sub drain tube' kept open. When the furnace reaches the temperature for CVD reaction, $\mathrm{Ar} / \mathrm{H}_{2}$ flow is stopped and the 'main drain tube' is opened to bring the inside of the quartz tube to vacuum. Subsequently, ethanol vapor is introduced into the quartz tube so that the pressure just before the entrance of the tube is constant at 10 Torr. After the CVD reaction, ethanol vapor is stopped and the furnace is turned off, before the apparatus is brought back to room temperature with the flow of $100 \mathrm{sccm}$ of $\mathrm{Ar} / \mathrm{H}_{2}$. 
The SWNTs in the as-grown state (i.e., without purification) were analyzed by FE-SEM (Hitachi, S-900), TEM (JEOL 2000EX), and micro-Raman scattering (Chromex 501is and Andor DV401-FI for the spectrometer and CCD system, respectively, using Seki Technotron STR250 optical system). All the Raman spectra shown in this report are an arithmetic average of 10 measurements performed at different locations on the substrate. VIS-NIR absorption spectra were measured using Hitachi U-4000 spectrophotometer.

\section{Results}

\subsection{Microscopic analyses}

Figure 2 shows a SEM image at a broken edge of the quartz substrate after $1 \mathrm{~h} \mathrm{CVD}$ at $800^{\circ} \mathrm{C}$. This image, similar to that in our previous Letter ${ }^{31)}$ but with improved visibility, was taken at an angle and includes the broken cross-section of the substrate. This image clearly shows the morphology of the SWNT mat directly synthesized on the quartz surface. SWNTs are randomly oriented and form bundles whose thickness mostly ranges between 7 - $15 \mathrm{~nm}$. On observing the edge, we were not able to find metallic agglomerates of more than $10 \mathrm{~nm}$ size that were often observed when catalytic metal was

mounted on the surface using sputtering or deposition ${ }^{22-25)}$. Detailed characterization of the metal catalyst on the surface is currently in progress by our group.

Although Fig. 2 yields mesoscopic information about the morphology, the microscopic structure as well as existence of contamination should be confirmed via TEM. We rubbed the blackened quartz surface with a microgrid and observed an edge of the SWNT mat adhered on the grid. Figure 3 shows an image observed with TEM. It was confirmed that only SWNTs were observed and no multi-walled nanotubes (MWNTs) were recognized. It was also confirmed that the wall of SWNTs was clean and almost free from amorphous carbon or any other impurities. As expected from the SEM observation, most of the SWNTs were observed to form bundles, however, some of the SWNTs were isolated as shown in Fig. 3.

\subsection{Raman scattering analyses}

Raman scattering analysis is a strong tool for characterizing obtained SWNTs ${ }^{32}$. The Raman spectra of SWNTs synthesized on $\mathrm{Si}$ and quartz substrates are presented in Fig. 4. Detailed interpretations of Raman spectra of SWNTs are referred to, e.g., ref. 33. The CVD reaction times were 10 and $60 \mathrm{~min}$ for Si and quartz, respectively. The distinct peak at approximately $1590 \mathrm{~cm}^{-1}$ (G-band) arises from an in-plane oscillation of carbon atoms in the sp2 graphene sheet and the less distinct peak at approximately $1350 \mathrm{~cm}^{-1}$ (D-band) reflects the degree of defects or dangling bonds contained in the sp2 arrangement of graphene. All the spectra were normalized by the height of the G-band peaks. 
For the case of Si substrates (Figs. 4(a)-4(c)), the amount of produced SWNTs is estimated from intensity ratio of the G-band and Si-derived peak at $960 \mathrm{~cm}^{-1}$. The amount of produced SWNTs markedly increases as the temperature increases; however, above $800^{\circ} \mathrm{C}$, the amount decreases again and no SWNTs were detected at $900^{\circ} \mathrm{C}$ (not shown). This is attributed to the silicide formation caused by the chemical reaction between catalyst metal and silicon, which significantly deteriorates catalytic activity as demonstrated by de los Arcos et al. ${ }^{34)}$. At any temperature, the produced SWNTs retain their high quality judging from its high G/D ratio of about 30. Most notably, with our method, SWNTs were synthesized on the Si surface even at $650^{\circ} \mathrm{C}^{31)}$ : This is by far the lowest temperature among the previous reports where $800^{\circ} \mathrm{C}^{25,27)}$ or $900^{\circ} \mathrm{C}$ (or higher) ${ }^{13-15,22-24)}$ was employed for SWNT synthesis. This technique is also effective for a quartz substrate and the spectrum (Fig. 4(d)) indicates that SWNTs with sufficiently high quality were obtained.

Figure 5 shows the Raman spectra of the 'radial breathing mode' (RBM) of SWNTs synthesized at $800^{\circ} \mathrm{C}$ grown on $\mathrm{Si}$ and quartz substrates [corresponding to Figs. 4(a) and 4(d), respectively] measured with three different laser wavelengths $488,514.5$, and $633 \mathrm{~nm}$. The RBM is unique to nanotubes and planar graphene does not present RBM peaks ${ }^{2}$. On the top of the figure, the Kataura plot ${ }^{35)}$ calculated using $\gamma_{0}=2.9 \mathrm{eV}$ and $\mathrm{a}_{\mathrm{c}-\mathrm{c}}=0.144 \mathrm{~nm}^{36)}$ is presented with horizontal lines corresponding to each laser energy. Since this is resonant Raman scattering, only limited SWNTs that have a band gap equal to the laser energy can resonantly scatter the incident laser. The peak locations at the bottom of Fig. 5 agree well with those predicted by the Kataura plot and it is recognized that the products for both cases were a mixture of both metallic and semiconducting nanotubes. Since it is known that the diameter of an SWNT is inversely proportional to the RBM shift ${ }^{33)}$, the diameter distribution is estimated using, e.g., the relationship ' $d(\mathrm{~nm})=248 / v\left(\mathrm{~cm}^{-1}\right)^{37)}$, where $d$ and $v$ are the diameter and Raman shift of a SWNT, respectively. From Fig. 5, it is recognized that SWNTs grown on Si and quartz surfaces have a diameter distribution of 1.1-1.7 nm, which coincides with the TEM observation in Fig. 3.

\section{Discussion}

\subsection{Effect of reduction}

We supplied $\mathrm{H}_{2}$ during heating of the electric furnace for the purpose of reduction of catalyst metals. Since the catalyst before the CVD is oxidized and therefore has less activity, one should reduce the catalyst by some means before SWNTs start to grow. Actually, since carbon itself is a good reducer, it is possible to reduce the metal oxides using alcohol vapor. However, since in this case the decomposition speed of alcohol to extract carbon atoms is considered to be slow due to the lower catalytic activity, the amount of SWNTs when the catalyst is not reduced prior to the CVD reaction is generally low. This was demonstrated in our previous report using zeolite as a support material ${ }^{12)}$ This 
discussion actually holds also in the case of a flat substrate. Figure 6 shows the Raman spectra taken at $488 \mathrm{~nm}$ from the surface of the Si substrate after CVD at $750^{\circ} \mathrm{C}$. Figure 6(a) is the spectrum when $\mathrm{Ar} / \mathrm{H}_{2}$ (3\% $\mathrm{H}_{2}$ ) is supplied during heat-up of the electric furnace, and Fig. 6(b) is the spectrum when Ar is used instead of $\mathrm{Ar} / \mathrm{H}_{2}$. The intensity of the G-band shown in Fig. 6(a) is about 40 times larger than that in Fig. 6(b), even though the reaction time in Fig. 6(b) was longer than that in Fig. 6(a). This indicates that the amount of SWNTs can be significantly controlled by the use of reduction besides changing the duration of CVD to control the amount of SWNTs.

\subsection{Effect of metallic salt}

We have employed bimetallic Mo/Co catalyst that exhibits satisfactory performance, as presented thus far. To date, various kinds of bimetallic combinations such as $\mathrm{Fe} / \mathrm{Co}^{11,12)}, \mathrm{Mo} / \mathrm{Co}^{38,39)}$, and $\mathrm{Mo} / \mathrm{Fe}^{6,8,9,40)}$ impregnated on support powder (e.g., silica, alumina, or MgO.) were employed for the mass synthesis of SWNTs. In some of these studies ${ }^{8,38-40)}$ the effect of bimetals for an enhancement in SWNT production was discussed.

Figure 7 shows Raman spectra (taken by $488 \mathrm{~nm}$ ) of SWNTs grown on quartz substrates with CVD at $800^{\circ} \mathrm{C}$ for $1 \mathrm{~h}$ using various catalysts. The CVD and Raman scattering measurement for the samples in Figs. 7(a)-7(d) were performed in the same batch to ensure comparability. In Fig. 7(a), our standard acetic Mo/Co catalyst (0.01 wt\% each) was used and the spectrum exhibited strong G-band peak at $1590 \mathrm{~cm}^{-1}$, as shown before. In the case shown in Fig. 7(b), we used a combination of molybdenum acetate and cobalt nitrate $\mathrm{Co}\left(\mathrm{NO}_{3}\right)_{2}-6 \mathrm{H}_{2} \mathrm{O}$. In this seemingly similar case to that shown in Fig. 7(a), however, the amount of SWNTs appreciably decreases from the case shown in Fig. 7(a): This may be explained by the detailed report by Sun et al. ${ }^{41)}$ who prepared cobalt catalysts on silica gel powder through an impregnation of mixture of cobalt (II) nitrate and cobalt (II) acetate salts. They tested various nitrate/acetate ratios from 1:0 (all nitrate) to 0:1 (all acetate) and demonstrated that only when acetate was solely used the fine dispersion of Co particles of approximately $2 \mathrm{~nm}$ in diameter were obtained, whereas when nitrate was used, the resultant Co particles became as large as $10 \mathrm{~nm}$ in diameter. They discussed that this was caused by a strong interaction between metal acetate and $\mathrm{SiO}_{2}$ support ${ }^{41}$. Although the microscopic structure of $\mathrm{SiO}_{2}$ in our case is different from that of ref. 41, the reduced catalytic activity in Fig. 7(b) might be caused by the weakened interaction between metals and the $\mathrm{SiO}_{2}$ surface. In the case of Fig. 7(c), a catalyst of Co $0.02 \mathrm{wt} \%$ was used and this resulted in a far weaker G-band intensity [1/25 of Fig. 7(a)]. Furthermore, when Mo 0.02 wt\% catalyst was employed [Fig. 7(d)] no signal of SWNTs was detected, indicating that monometallic Mo is inactive as a catalyst for SWNTs. Our observation here agrees with the discussion by Alvarez et al. ${ }^{39)}$ who explained the role of Mo as a stabilizer that prevents Co from agglomerating and therefore helps the catalyst in being suitable for 
SWNT formation. It is clear that the choice of Mo and Co acetates gives the best result among them; however, more detailed investigations on this matter are needed to clarify the effect of catalyst choice on the synthesis of SWNTs.

\subsection{Merits and application of current technique}

Since the generation of SWNTs requires an active catalyst with a size comparable to the diameter of a SWNT (i.e., a few nanometers) ${ }^{5)}$, it is critical to mount small amounts of catalytic metals on the surface at the same time to prevent them from agglomerating into large particles even under the high temperature in CVD. One obvious merit of our dip-coating technique lies in the ease of loading very small amounts of bimetallic catalyst on the surface partly because one can adjust the concentration of catalyst solution by diluting. In addition, it has been confirmed by TEM that the catalyst prepared by our method is finely dispersed over approximately 1-2 nanometers after heating with $\mathrm{Ar} / \mathrm{H}_{2}$ reduction ${ }^{41)}$. This good dispersion might be contributed by the strong chemical interaction between metal acetate and $\mathrm{SiO}_{2}$ base ${ }^{42)}$, as mentioned above. It is quite plausible that such chemical interaction does not occur in the catalyst loaded by sputtering or deposition processes.

Lowering of CVD temperature is essential because a higher temperature accelerates the sintering of catalysts and chemical reaction between catalyst metal and silicon to yield inactive metallic silicide $^{21,34)}$. The alcohol CCVD method employed in this report excels, as demonstrated in past reports $^{11,12,19)}$, in producing relatively high-quality SWNTs at low temperatures down to $600^{\circ} \mathrm{C}$. This low-temperature process contributes to our direct synthesis technique because, by this, we can be free from conventionally used support materials.

To the best of the authors' knowledge, this is the first study to directly synthesize visually recognizable amounts of SWNTs on the surface of a quartz substrate. Recently, it has been reported that SWNTs exhibit excellent bleached absorption properties due to their strong third-order optical non-linearity $\left(\chi^{(3)}\right)$ along with absorption of approximately $1.55 \mu \mathrm{m}$ that is used in optical telecommunication $^{43)}$. These characteristics motivated attempts to apply SWNTs toward optical switching $^{43)}$ and a mode-locked laser.

The SWNT-synthesized quartz substrate prepared by our technique could be used for such optical applications. An optical absorption spectrum of the specimen shown in Fig. 2 is presented in Fig. 8(a). As a reference, the spectrum of SWNTs synthesized on zeolite support using $\mathrm{Fe} / \mathrm{Co}$ catalyst ${ }^{12)}$ is presented in Fig. 8(b), which was suspended in 1 wt\% SDS-added $\mathrm{D}_{2} \mathrm{O}$ after strong sonication, centrifugation, and decantation of its supernatant based on the procedure reported in ref. 44. In the spectrum in Fig. 8(a), several broad peaks are recognized and this broadening is due to the bundling of SWNTs $^{45)}$. Sharper peaks are seen when bundles of SWNTs are removed by centrifugation (Fig. 8(b)). 
The difference in the locations of peaks between Figs. 8(a) and 8(b) comes from the difference in bandgap between contained SWNTs (i.e. difference in chirality) in addition to a red shift of the spectrum shown in Fig. 8(a) due to bundling. There are some recognizable groups of peaks in the spectra. The peaks at approximately $1.45 \mu \mathrm{m}$ in Fig. 8(a) are derived from the first-energy gaps of semiconducting tubes $E_{11}^{S}$, which corresponds to the peaks around $1.3 \mu \mathrm{m}$ in the case of Fig. 8(b), and the peaks at approximately $800 \mathrm{~nm}$ from the second-energy gaps $E_{22}^{S}{ }^{45}$. These results verify the possibility of application of the current technique to the aforementioned optical applications. To date, we have been able to synthesize SWNTs directly on the surface of an optical fiber using the same technique, which may also contribute to such SWNT-based optical technologies ${ }^{46)}$.

\section{Conclusion}

We have presented a method of synthesizing SWNTs with sufficient quality directly on the surface of Si and quartz substrates using the dip-coating method for catalyst loading and ACCVD method for the CVD process. The SWNTs were synthesized directly on a Si surface even at the temperature of $650^{\circ} \mathrm{C}$, which is far lower than the reported cases of between 800 to $1000^{\circ} \mathrm{C}$. In the case of quartz, abundant amounts of SWNTs were produced on the surface to the extent that the surface was blackened with SWNTs. The proposed dip-coating method is an easy and cost-effective approach that is suitable for mounting small amounts of catalysts on the surface. The strong interaction between metal acetate and $\mathrm{SiO}_{2}$ base may aid the fine dispersion of catalyst that is sometimes difficult to achieve by conventional sputtering and deposition techniques, though further elucidation of the catalyst formation process is essential. This technique may be applicable to recently proposed SWNT-based optical devices.

\section{Acknowledgements}

The authors thank Mr. H. Tsunakawa for his assistances with the TEM operation and T. Sugawara for his advices in SEM operation, both at the University of Tokyo. Part of this work was financially supported by KAKENHI \#13555050 from JSPS, and \#13GS0019 from MEXT. This paper was originally presented at International Symposium on Micro-Mechanical Engineering 2003 (ISMME 2003), held in Tsukuba, Japan. 
Jpn. J. Appl. Phys. in press

\section{References}

[1] S. Iijima and T. Ichihashi: Nature 363 (1993) 603.

[2] R. Saito, G. Dresselhaus and M. S. Dresselhaus: Physical Properties of Carbon Nanotubes (Imperial College Press, London, 1998).

[3] A. Thess, R. Lee, P. Nikolaev, H. Dai, P. Petit, J. Robert, C. H. Xu, Y. H. Lee, S. G. Kim, A. G. Rinzler, D. T. Colbert, G. E. Scuseria, D. Tomanek, J. E. Fischer and R. E. Smalley: Science 273 (1996) 483.

[4] C. Journet, W. K. Maser, P. Bernier, A. Loiseau, M. L. Chapelle, S. Lefrant, P. Deniard, R. Lee and J. E. Fischer: Nature 388 (1997) 756.

[5] H. Dai, A. G. Rinzler, P. Nikolaev, A. Thess, D. T. Colbert and R. E. Smalley: Chem. Phys. Lett. 260 (1996) 471.

[6] J. H. Hafner, M. J. Bronikowski, B. R. Azamian, P. Nikolaev, A. G. Rinzler, D. T. Colbert, K. A. Smith and R. E. Smalley: Chem. Phys. Lett. 296 (1998) 195.

[7] P. Nikolaev, M. J. Bronikowski, R. K. Bradley, F. Rohmund, D. T. Colbert, K. A. Smith and R. E. Smalley: Chem. Phys. Lett. 313 (1999) 91.

[8] A. M. Cassell, J. A. Raymakers, J. Kong and H. Dai: J. Phys. Chem. B. 103 (1999) 6484.

[9] M. Su, B. Zheng and J. A. Liu: Chem. Phys. Lett. 322 (2000) 321.

[10] J. F. Colomer, J. M. Benoit, C. Stephan, S. Lefrant, G. Van Tendeloo and J. B. Nagy: Chem. Phys. Lett. 345 (2001) 42.

[11] S. Maruyama, R. Kojima, Y. Miyauchi, S. Chiashi and M. Kohno: Chem. Phys. Lett. 360 (2002) 229.

[12] Y. Murakami, Y. Miyauchi, S. Chiashi and S. Maruyama: Chem. Phys. Lett. 374 (2003) 53.

[13] J. Kong, H. T. Soh, A. M. Cassell, C. F. Quate and H. Dai: Nature 395 (1998) 878.

[14] N. R. Franklin, Q. Wang, T. W. Tombler, A. Javey, M. Shim and H. Dai: Appl. Phys. Lett. 81 (2002) 913.

[15] A. M. Cassell, N. R. Franklin, T. W. Tombler, E. M. Chan, J. Han and H. Dai: J. Am. Chem. Soc. 121 (1999) 7975.

[16] A. M. Cassell, S. Verma, L. Delzeit, M. Meyyappan and J. Han: Langmuir 17 (2001) 260.

[17] G. Zheng, H. Zhu, Q. Luo, Y. Zhou and D. Zhao: Chem. Mater. 13 (2001) 2240.

[18] N. Petkov, S. Mintova, K. Karaghiosoff and T. Bein: Mat. Sci. Eng. C 23 (2003) 145.

[19] Y. Murakami, S. Yamakita, T. Okubo and S. Maruyama: Chem. Phys. Lett. 375 (2003) 393.

[20] Y. Li, W. Kim, Y. Zhang, M. Rolandi, D. Wang and H. Dai: J. Phys. Chem. B 105 (2001) 11424.

[21] Y. Homma, T. Ymashita, P. Finnie, M. Tomita and T. Ogino: Jpn. J. Appl. Phys 41 (2002) L89.

[22] O. A. Nerushev, R. E. Morjan, D. I. Ostrovskii, M. Sveningsson, M. Jonsson, F. Rohmund and E. E. 
B. Campbell: Physica B 323 (2002) 51.

[23] Y. J. Yoon, J. C. Bae, H. K. Baik, S. Cho, S. J. Lee, K. M. Song and N. S. Myung: Chem. Phys. Lett. 366 (2002) 109.

[24] L. Delzeit, B. Chen, A. Cassell, R. Stevens, C. Nguyen and M. Meyyappan: Chem. Phys. Lett. 348 (2001) 368.

[25] R. Y. Zhang, L. Amlani, J. Baker, J. Tresek and R. K. Tsui: Nano Lett. 3 (2003) 731.

[26] H. Hongo, M. Yudasaka, T. Ichihashi, F. Nihey and S. Iijima: Chem. Phys. Lett. 361 (2002) 349.

[27] Y. J. Jung, Y. Homma, T. Ogino, Y. Kobayashi, D. Takagi, B. Wei, R. Vajtai and P. M. Ajayan: J. Phys. Chem. B 107 (2003) 6859.

[28] K. Matsumoto, S. Kinoshita, Y. Gotoh, K. Kurachi, T. Kamimura, M. Maeda, K. Sakamoto, M. Kuwahara, N. Atoda and Y. Awano: Jpn. J. Appl. Phys. 42 (2003) 2415.

[29] Y. Ohno, S. Iwatsuki, T. Hiraoka, T. Okazaki, S. Kishimoto, K. Maezawa, H. Shinohara and T. Mizutani: Jpn. J. Appl. Phys. 42 (2003) 4116.

[30] F. Nihey, H. Hongo, M. Yudasaka and S. Iijima: Jpn. J. Appl. Phys. 41 (2002) L1049.

[31] Y. Murakami, Y. Miyauchi, S. Chiashi and S. Maruyama: Chem. Phys. Lett. 377 (2003) 49.

[32] A. M. Rao, E. Richter, S. Bandow, B. Chase, P. C. Eklund, K. A. Williams, S. Fang, K. R. Subbaswamy, M. Menon, A. Thess, R. E. Smalley, G. Dresselhaus and M. S. Dresselhaus: Science 275 (1997) 187.

[33] M. S. Dresselhaus and P. C. Eklund: Adv. Phys. 49 (2000) 705.

[34] T. de los Arcos, F. Vonau, M. G. Garnier, V. Thommen, H. G. Boyen, P. Oelhafen, M. Duggelin, D. Mathis and R. Guggenheim: Appl. Phys. Lett. 80 (2002) 2383.

[35] H. Kataura, Y. Kumazawa, Y. Maniwa, I. Umezu, S. Suzuki, Y. Ohtsuka and Y. Achiba: Synth. Met. 103 (1999) 2555.

[36] R. Saito, G. Dresselhaus and M. S. Dresselhaus: Phys. Rev. B 61 (2000) 2981.

[37] A. Jorio, R. Saito, J. H. Hafner, C. M. Lieber, M. Hunter, T. McClure, G. Dresselhaus and M. S. Dresselhaus: Phys. Rev. Lett. 86 (2001) 1118.

[38] B. Kitiyanan, W. E. Alvarez, J. H. Harwell and D. E. Resasco: Chem. Phys. Lett. 317 (2000) 497.

[39] W. E. Alvarez, B. Kitiyanan, A. Borgna and D. E. Resasco: Carbon 39 (2001) 547.

[40] A. R. Harutyunyan, B. K. Pradhan, U. J. Kim, G. G. Chen and P. C. Eklund: Nano Lett. 2 (2002) 525.

[41] M. Hu, Y. Murakami, M. Ogura, S. Maruyama and T. Okubo: J. Phys. Chem. B, submitted.

[42] S. Sun, N. Tsubaki and K. Fujimoto: Appl. Catal. A 202 (2002) 121.

[43] S. Tatsuura, M. Furuki, Y. Sato, I. Iwasa, M. Q. Tian and H. Mitsu: Adv. Mater. 15 (2003) 534.

[44] S. Maruyama, Y. Miyauchi, Y. Murakami and S. Chiashi: New J. Phys. 5 (2003) 149.1. 
Jpn. J. Appl. Phys. in press

[45] M. J. O'Connell, S. M. Bachilo, C. B. Huffman, V. C. Moore, M. S. Strano, E. H. Haroz, K. L. Rialon, P. J. Boul, W. H. Noon, C. Kittrell, J. P. Ma, R. H. Hauge, R. B. Weisman and R. E. Smalley: Science 297 (2002) 5936.

[46] S. Yamashita, S. Maruyama, Y. Murakami, Y. Inoue, H. Yaguchi, M. Jablonski and S.Y.Set: The 9th Microoptics Conference, Tokyo, Japan, 2003. 


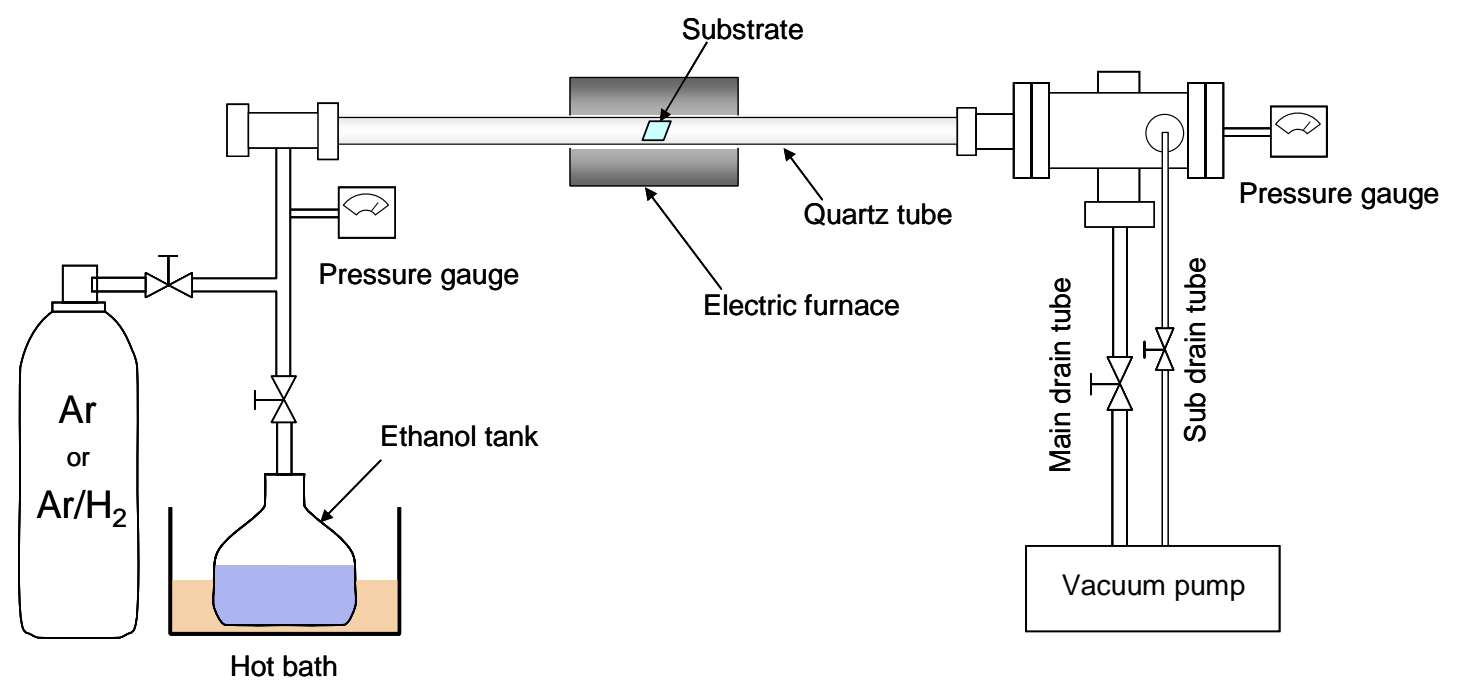

Fig. 1. Schematic of our CVD apparatus for synthesis of SWNTs. 


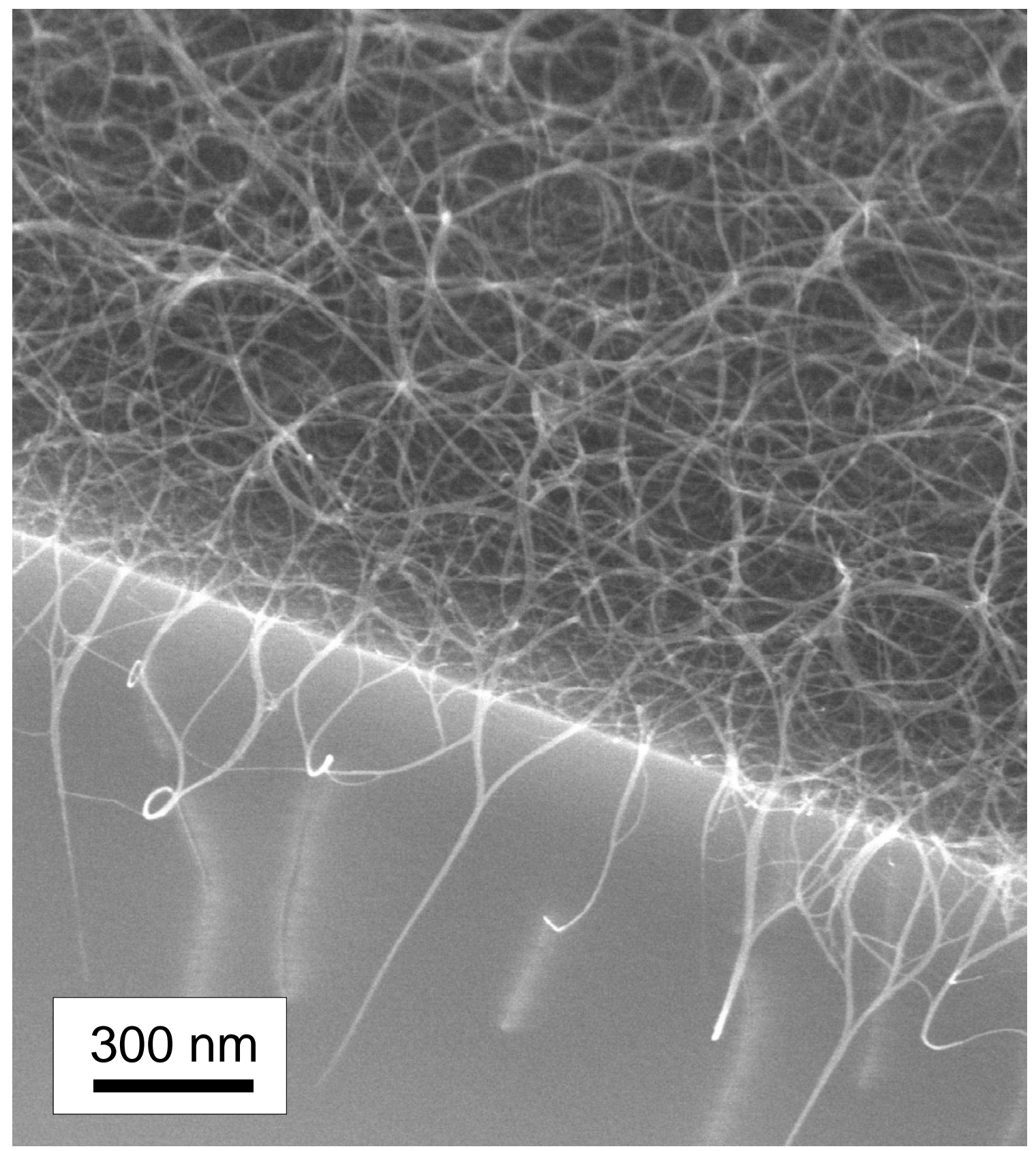

Fig. 2. SEM image of SWNT mat directly synthesized on a quartz substrate. Lower half is the broken cross-section of the substrate. CVD conditions were $800{ }^{\circ} \mathrm{C}$ and $1 \mathrm{~h}$ for reaction temperature and time, respectively. 


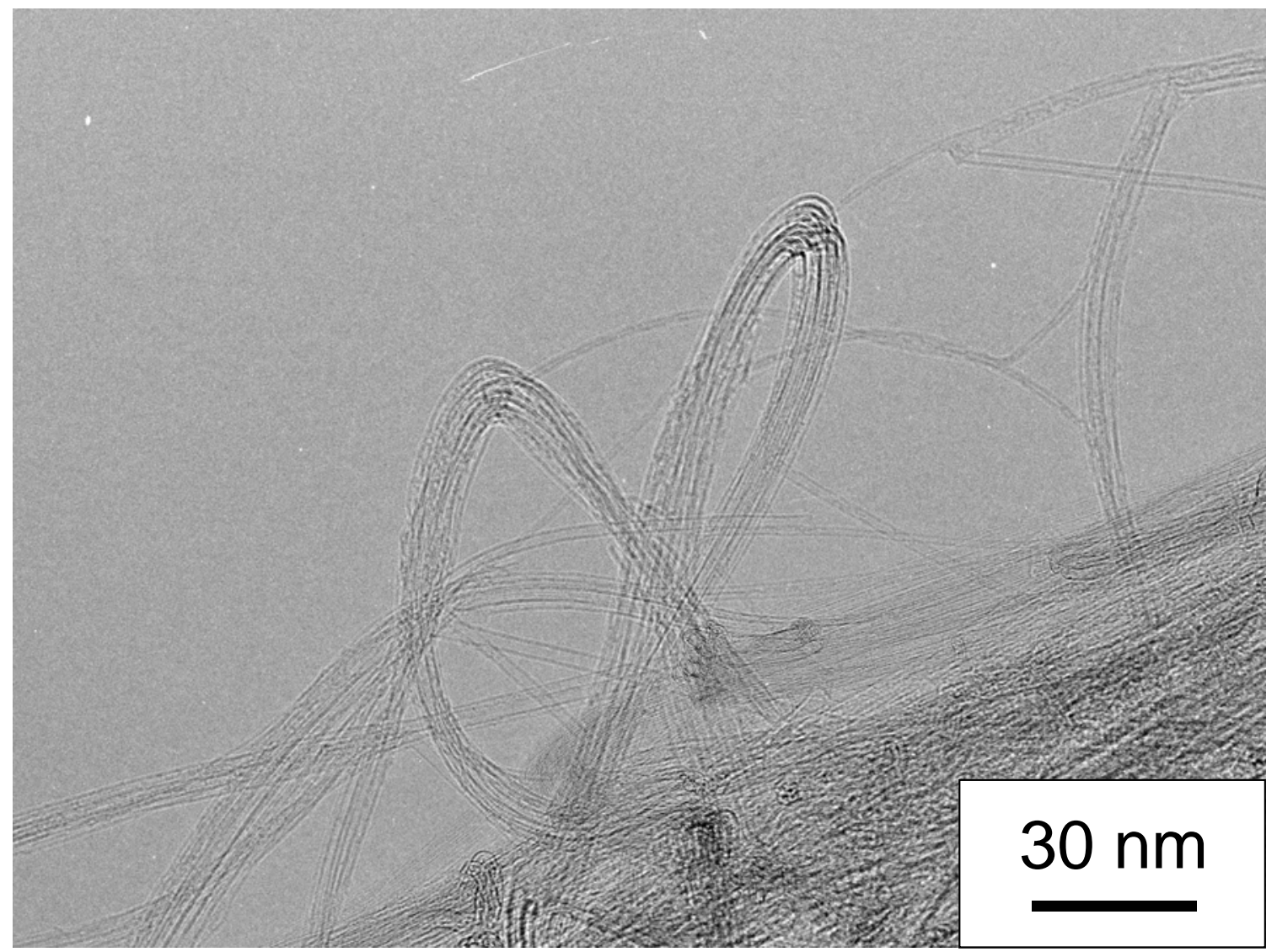

Fig. 3. TEM observations of SWNTs shown in Fig. 2. An edge of the SWNT mat was observed. Lower right corner corresponds to the thick mat where the electron transmission is weak and therefore appears darkened. 


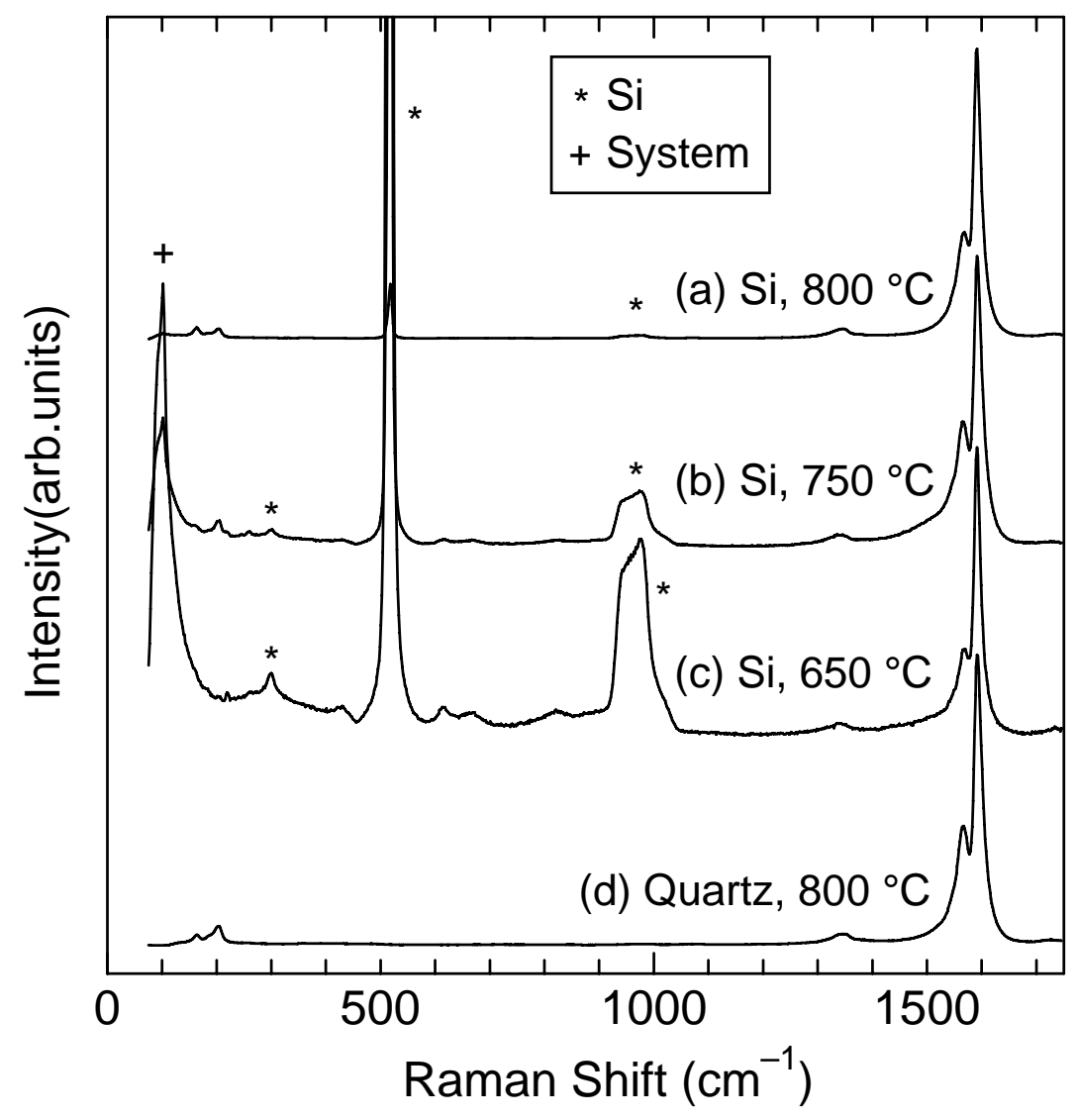

Fig. 4. Raman spectra (taken by $488 \mathrm{~nm}$ ) of SWNTs grown directly on the Si and quartz substrates under different CVD temperatures. CVD times for Si and quartz are $10 \mathrm{~min}$ and $1 \mathrm{~h}$, respectively. 


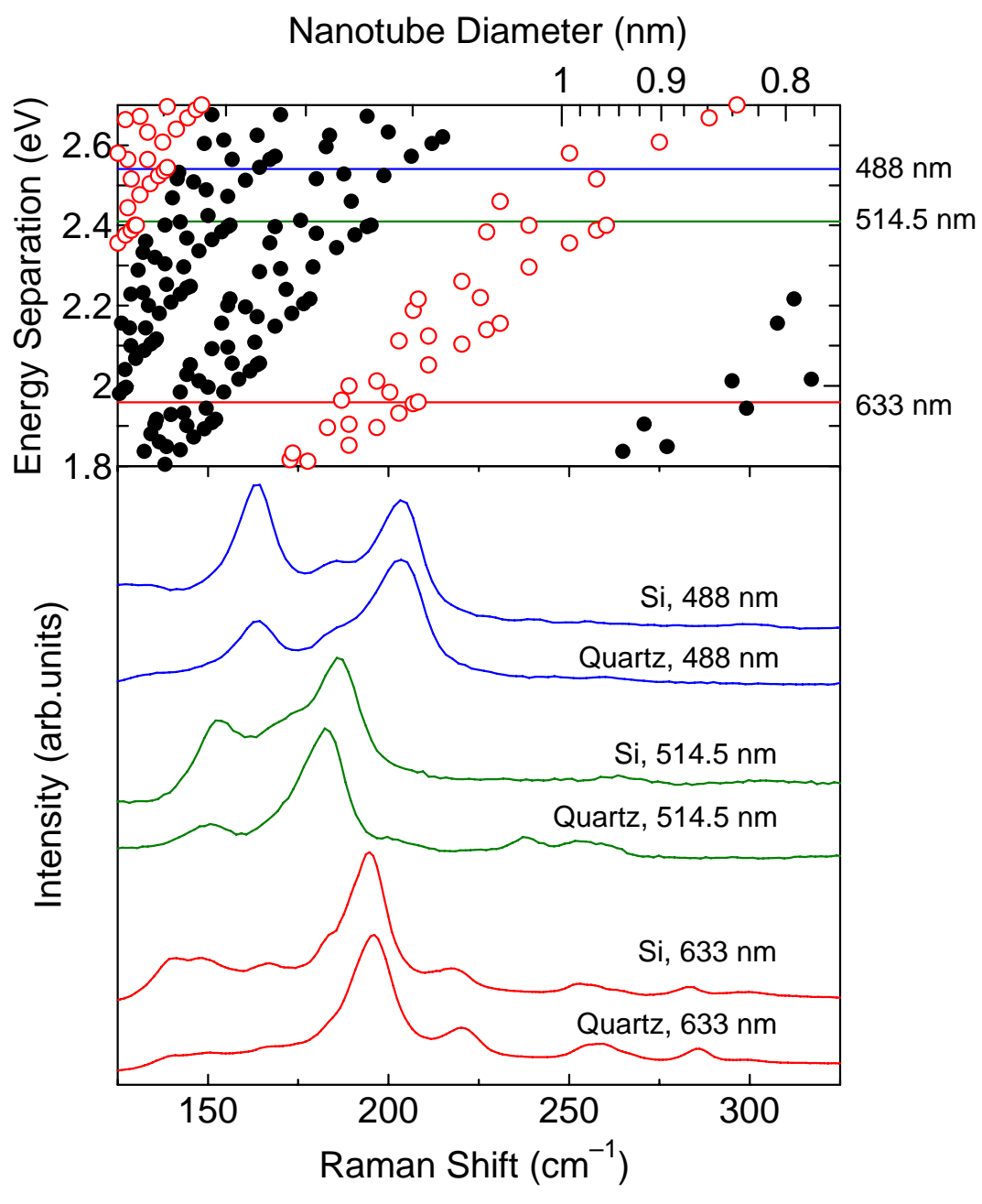

Fig. 5. RBM spectra of SWNTs grown on Si and quartz substrates taken by three different lasers, 488, 514.5, and $633 \mathrm{~nm}$. On the top is the Kataura plot where open and solid circles denote the energy gaps of metallic and semiconducting SWNTs, respectively. Horizontal lines denote energies of incident lasers. 


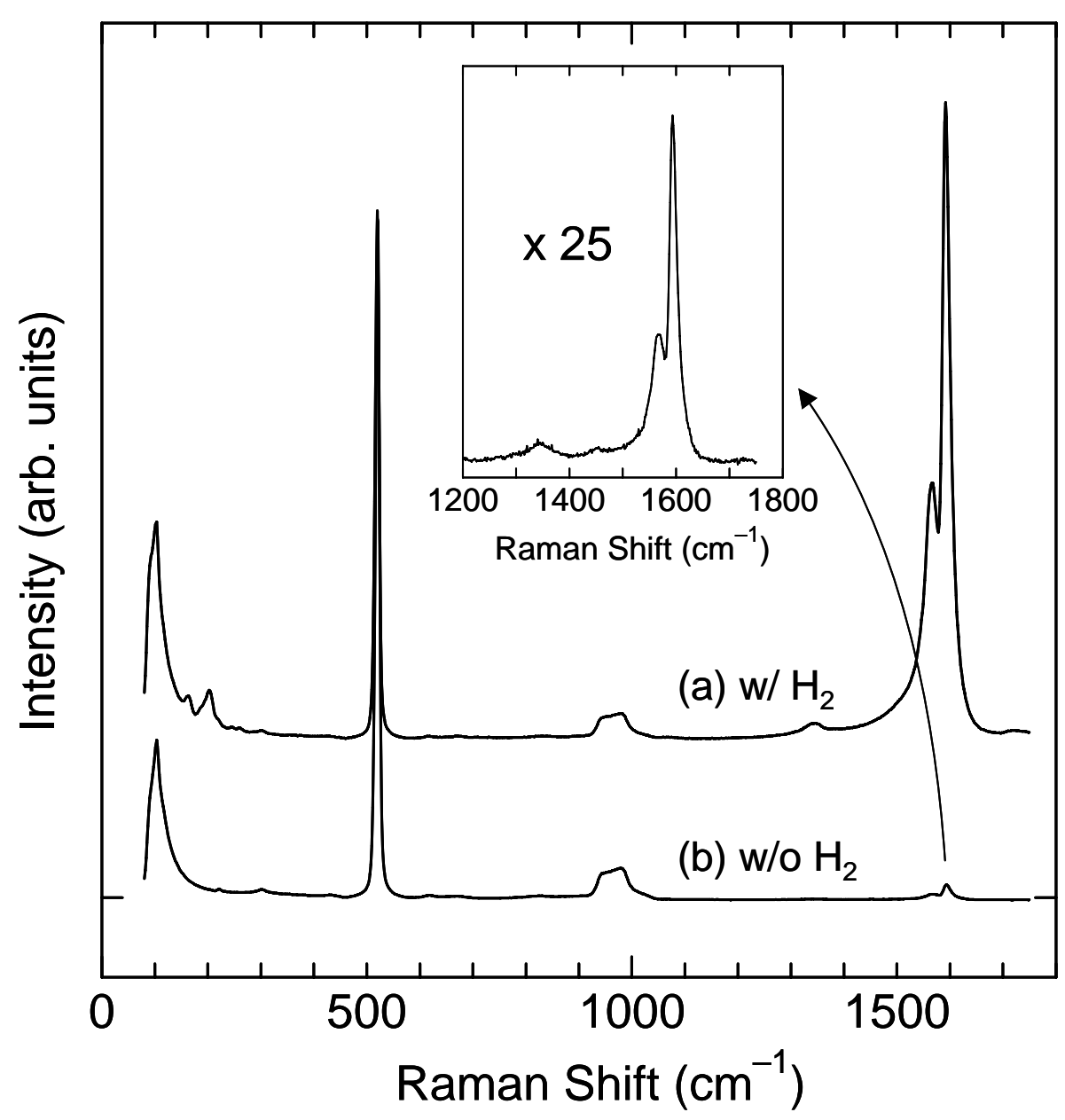

Fig. 6. Raman spectra (taken by $488 \mathrm{~nm}$ ) of SWNTs synthesized on Si surface when (a) $\mathrm{Ar} / \mathrm{H}_{2}\left(3 \% \mathrm{H}_{2}\right.$ ) or (b) Ar was flowed during heat-up of the electric furnace. CVD was performed at $750{ }^{\circ} \mathrm{C}$ for (a) 10 min and (b) 30 min. Inset magnifies G-band in the case of (b). CVD times for Si and quartz are $10 \mathrm{~min}$ and $1 \mathrm{~h}$, respectively. 

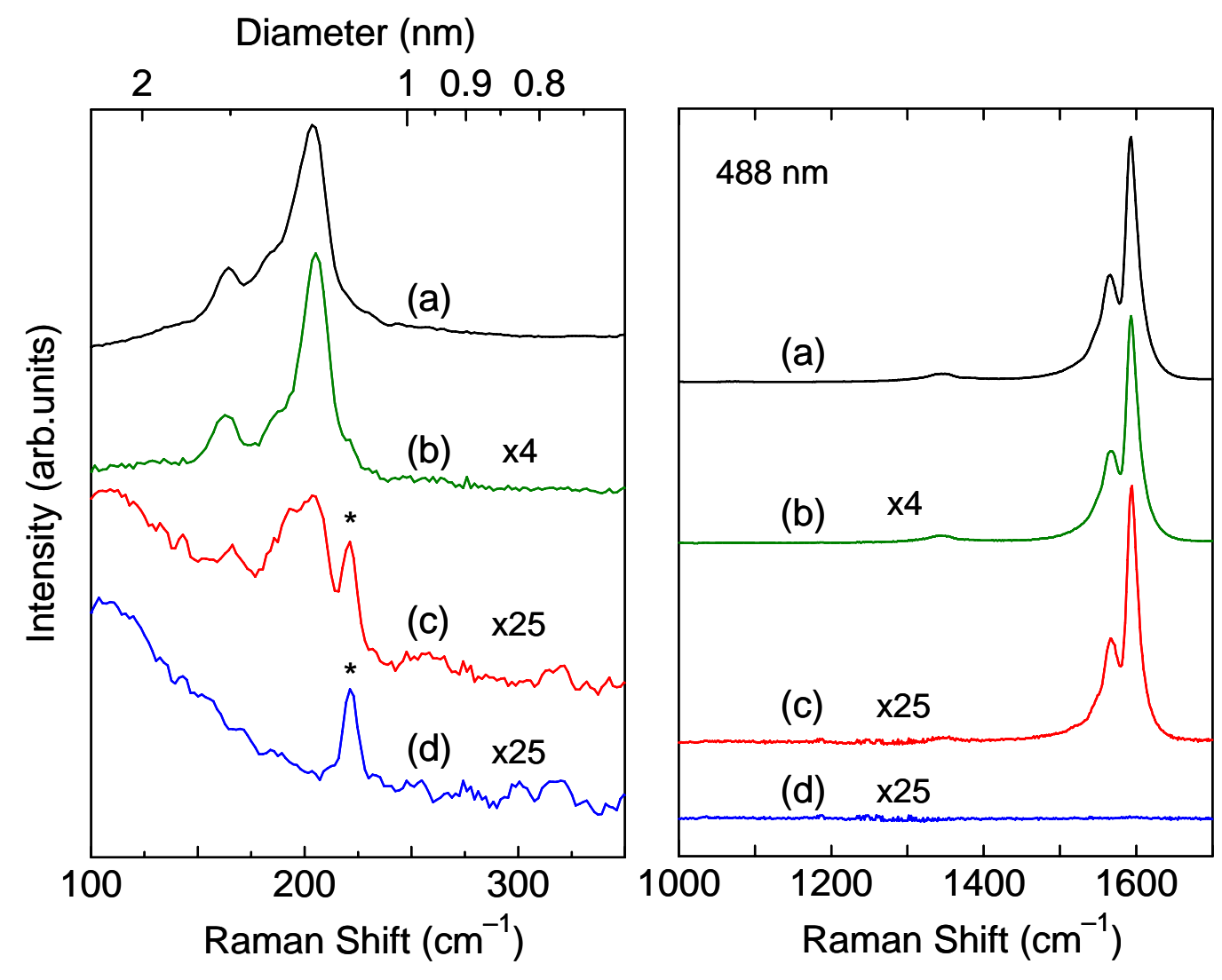

Fig. 7. Raman spectra (taken by $488 \mathrm{~nm}$ ) of SWNTs generated on quartz substrates from various catalysts, (a) Co acetate and Mo acetate $0.01 \mathrm{wt} \%$ each, (b) Co nitrate and Mo acetate $0.01 \mathrm{wt} \%$ each, (c) Co acetate $0.02 \mathrm{wt} \%$, and (d) Mo acetate $0.02 \mathrm{wt} \%$. The weight concentration here is defined as the ratio of metallic weight in salt to total solution weight used for the dip-coating process (see experimental section). The right panel shows G-band while left panel exhibits RBM. The asterisk at $220 \mathrm{~cm}^{-1}$ denotes the background noise of our measurement system. 


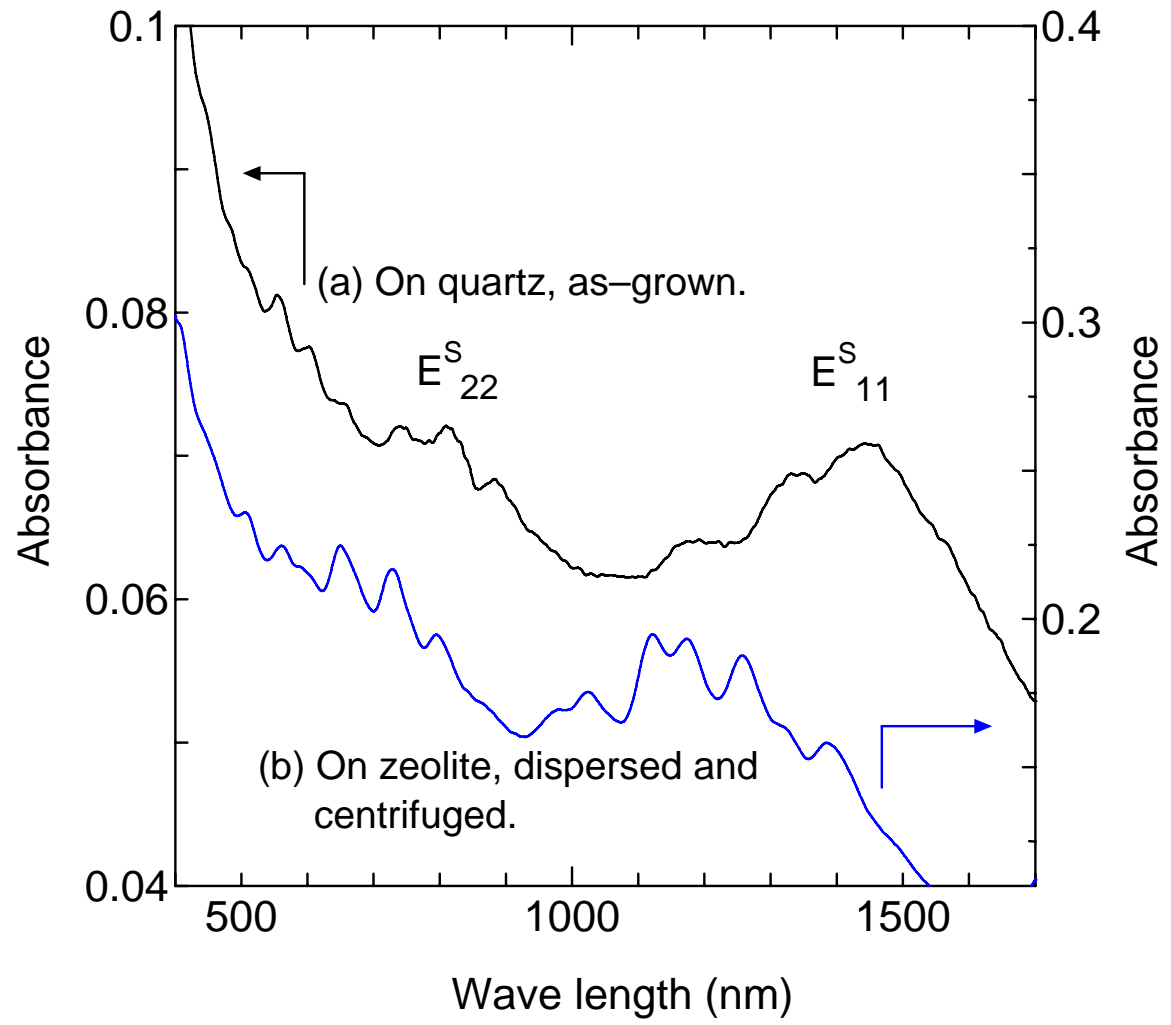

Fig. 8. Optical absorption spectrum of (a) SWNTs synthesized directly on the surface of quartz, which corresponds to the sample shown in Figs. 2 and 3, and (b) those synthesized on zeolite support using $\mathrm{Fe} / \mathrm{Co}$ catalyst ${ }^{12)}$ as a reference. The spectrum in the case of (b) was measured by dispersing the specimen in 1 wt\% SDS-added $\mathrm{D}_{2} \mathrm{O}$ solution followed by a strong sonication, centrifugation, and decantation of its supernatant based on the procedure in ref. 44 . 